\title{
Land of hope and dreams
}

\section{Selection of life science and translational medicine literature \\ by Marco Confalonieri}

Telomeres serve as protective "end caps" for chromosomes, help keep DNA healthy and functioning as it can replicate. These protective end caps become shorter with each DNA replication, and eventually are not longer able to protect DNA from sustaining damage and mutations, when cells become senescent. A possible way to produce cell rejuvenation might be to extend telomeres leading to longer and healthier cell life. Researchers from the Institute for Stem Cell Biology and Regenerative Medicine at Stanford University have taken an important step toward solving this problem by developing a new treatment used in the laboratory that extends telomeres. Have they got the Holy Grail of regenerative medicine finding a method to reverse cellular senescence and the main mechanism responsible for the finite proliferative capacity of somatic cells? The paper published this year on FASEB journal shows how to transfect fibroblasts and myofibroblasts delivering into the cells mRNA encoding telomerase reverse transcriptase (TERT). This catalytic subunit of the enzyme telomerase, together with the telomerase RNA component TERC, is the main component of the telomerase complex that lengthens telomeres in DNA strands, thereby allowing senescent cells to become potentially immortal. TERT catalyzes the addition of nucleotides in a TTAGGG DNA sequence to the ends of a chromosome's telomeres preventing degradation of the chromosomal ends following multiple rounds of replication. Hopefully, $\mathrm{He}$ len Blau and her colleagues found a historical important method to reverse chronic degenerative diseases and/or genetic telomerase disorders, but it is too early for therapeutic implications ready for medical practice. Deregulation of telomerase expression may be involved in the development of many cancers, including lung cancer. Nevertheless, this study reassured about the potential safety of this new approach to extend telomeres showing that the transfected cells were not immortalized and the telomeres resumed shortening after the short transient treatment with $\mathrm{mR}$ NA encoding TERT.

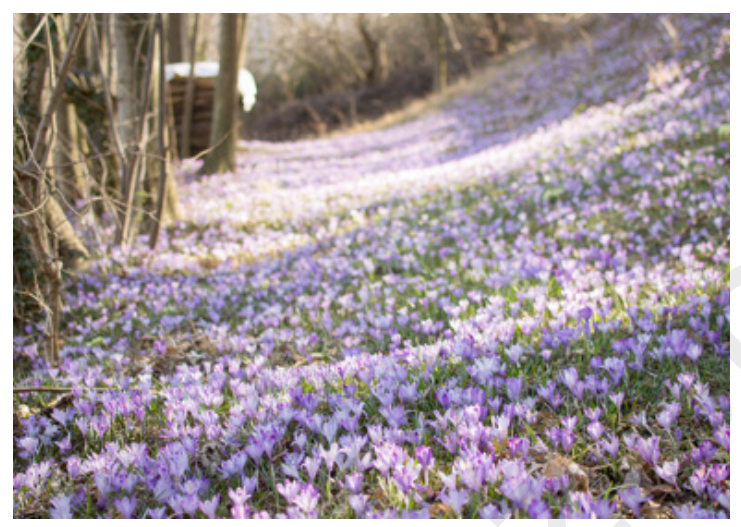

1) Transient delivery of modified mRNA encoding TERT rapidly extends telomeres in human cells Ramunas J., Yakubov E., Brady J.J., Corbel S.Y., Holbrook C., Brandt M., Stein J., Santiago J.G., Cooke J.P., Blau H.M.

FASEB J 2015 Jan 22;pii:fj. 14-259531

\section{Abstract}

Telomere extension has been proposed as a means to improve cell culture and tissue engineering and to treat disease. However, telomere extension by nonviral, nonintegrating methods remains inefficient. Here we report that delivery of modified mRNA encoding TERT to human fibroblasts and myoblasts increases telomerase activity transiently (24-48 h) and rapidly extends telomeres, after which telomeres resume shortening. Three successive transfections over a $4 \mathrm{~d}$ period extended telomeres up to $0.9 \mathrm{~kb}$ in a cell type-specific manner in fibroblasts and myoblasts and conferred an additional $28 \pm 1.5$ and $3.4 \pm 0.4$ population doublings (PD), respectively. Proliferative capacity increased in a dose-dependent manner. The second and third transfections had less effect on proliferative capacity than the first, revealing a refractory period. However, the refractory period was transient as a later fourth transfection increased fibroblast proliferative capacity by an additional $15.2 \pm 1.1 \mathrm{PD}$, similar to the first transfection. Overall, these treatments led to an increase in absolute cell number of more than 1012-fold. Notably, unlike immortalized cells, all treated cell populations eventually stopped increasing in number and expressed senescence markers to the same extent as untreated cells. This rapid method of extending telomeres and increasing cell proliferative capacity without risk of insertional mutagenesis should have broad utility in disease modeling, drug screening, and regenerative medicine. 